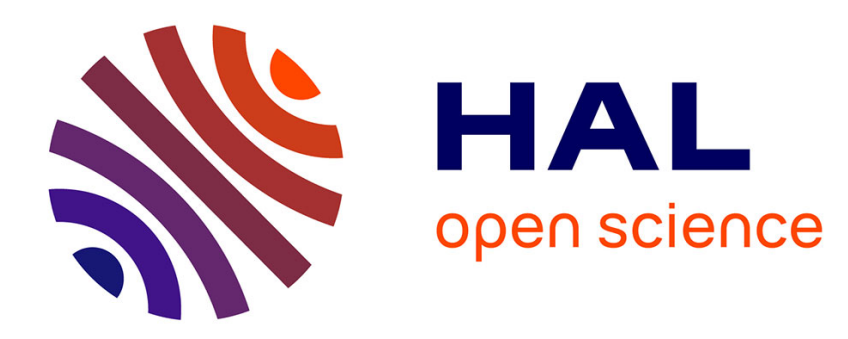

\title{
Supersymmetry and theory of heavy fermions
}

\author{
C. Pépin, M. Lavagna
}

\section{To cite this version:}

C. Pépin, M. Lavagna. Supersymmetry and theory of heavy fermions. Physical Review B: Condensed Matter and Materials Physics (1998-2015), 1999, 59 (19), pp.12180 - 12183. 10.1103/PhysRevB.59.12180 . hal-01896146

\section{HAL Id: hal-01896146 \\ https://hal.science/hal-01896146}

Submitted on 16 Oct 2018

HAL is a multi-disciplinary open access archive for the deposit and dissemination of scientific research documents, whether they are published or not. The documents may come from teaching and research institutions in France or abroad, or from public or private research centers.
L'archive ouverte pluridisciplinaire HAL, est destinée au dépôt et à la diffusion de documents scientifiques de niveau recherche, publiés ou non, émanant des établissements d'enseignement et de recherche français ou étrangers, des laboratoires publics ou privés. 


\title{
Supersymmetry and theory of heavy fermions
}

\author{
C. Pépin* and M. Lavagna \\ Commissariat à l'Energie Atomique, Département de Recherche Fondamentale sur la Matière Condensée /SPSMS, \\ 17, rue des Martyrs, 38054 Grenoble Cedex 9, France
}

(Received 4 February 1999)

\begin{abstract}
We propose an approach to the Kondo lattice in order to describe simultaneously the Kondo effect and the local magnetism. This approach relies on an original representation of the $S=1 / 2$ impurity spin in which the different degrees of freedom are represented by fermionic as well as bosonic variables. The band structure shows a bosonic mode in addition to the usual fermionic bands found in slave-boson theories. The density of states at the Fermi level is strongly enhanced and the Fermi-surface sum rule includes $n_{c}+1$ states. Finally, we study the dynamical spin susceptibility and the optical conductivity. [S0163-1829(99)04719-0]
\end{abstract}

An outstanding feature of heavy-Fermion systems is the coexistence of Fermi-liquid-type excitations with local magnetism resulting from Ruderman-Kittel-Kasuya-Yosida (RKKY) interactions among spins as shown by a number of experiments. An important probe is provided by the de Haasvan Alphen (dHvA) experiments. The results ${ }^{1}$ indicate heavy effective mass. They also agree as to the existence of large Fermi surfaces in the magnetically-disordered phase. Even though the charge degrees of freedom are frozen, the localized electrons seem to contribute to the Fermi-surface sum rule together with the conduction electrons.

This coexistence of Fermi-liquid-type excitations with low energy magnetic fluctuations is likely to stem from the nature of the screening of the localized moments in the Kondo lattice. According to the exhaustion principle, at $T_{K}$ the available conduction electrons are exhausted ${ }^{2}$ before achieving complete screening (incomplete Kondo effect) leaving residual unscreened spin degrees of freedom on the impurities.

Traditionally, the spin is described either in fermionic or bosonic representation. If the former representation, used for instance in the $1 / N$ expansion of the Anderson $^{3}$ or the Kondo ${ }^{4}$ lattice, appears to be well adapted for the description of the Kondo effect, it is also clear that the bosonic representation lends itself better to the study of local magnetism. Quite obviously the physics of heavy Fermions is dominated by the duality between Kondo effect and localized moments. This constitutes our motivation to introduce an approach to the Kondo lattice model (KLM) that relies on an original representation of the impurity spin $1 / 2$ in which the different degrees of freedom are represented by fermionic as well as bosonic variables. The former are believed to describe the Fermi-liquid excitations while the latter account for the residual spin degrees of freedom.

Let us list the main results obtained in this paper: (i) the band structure shows a bosonic band in addition to the fermionic bands of the standard slave-boson theories; the density of states at the Fermi level is strongly enhanced, (ii) the Fermi-surface sum rule includes $n_{c}+1$ states, which means that the Fermi-surface volume includes a contribution of one state per localized spin in agreement with dHvA experiments, (iii) the dynamical susceptibility is dominated by the presence of the bosonic band while the optical conductivity is not; the gap appearing in the frequency dependence of the optical conductivity is equal to the direct gap between the two fermionic bands.

In order to include the Fermi-liquid excitations as well as the residual spin degrees of freedom, we propose to enlarge the representation of the spin operator as follows

$$
S^{a}=\sum_{\sigma \sigma^{\prime}} b_{\sigma^{\dagger}}^{\dagger} \tau_{\sigma \sigma^{\prime}}^{a} b_{\sigma^{\prime}}+f_{\sigma}^{\dagger} \tau_{\sigma \sigma^{\prime}}^{a} f_{\sigma^{\prime}}=S_{b}^{a}+S_{f}^{a},
$$

where $b_{\sigma}^{\dagger}$ and $f_{\sigma}^{\dagger}$ are, respectively, bosonic and fermionic creation operators and $\tau^{a}[a=(+,-, z)]$ are Pauli matrices. Equation (1) corresponds to a mixed fermionic-bosonic representation between Schwinger bosons and Abrikosov pseudofermions. An additional constraint needs to be introduced in order to complete the representation

$$
n_{f}+n_{b}=1
$$

This constraint can be viewed as a charge conservation of the following $S U(1 \mid 1)$ fermion-boson rotation symmetry leaving the spin operator invariant

$$
\left(f_{\sigma}^{\prime \dagger}, b_{\sigma}^{\prime \dagger}\right)=\left(f_{\sigma}^{\dagger}, b_{\sigma}^{\dagger}\right) V^{\dagger}
$$

where $V^{\dagger}$ is an unitary supersymmetric matrix $\left(V V^{\dagger}=V^{\dagger} V\right.$ $=1)$. It is important to notice that this representation overcounts the states. Four states $f_{\uparrow}^{\dagger}|0\rangle, f_{\downarrow}^{\dagger}|0\rangle, b_{\uparrow}^{\dagger}|0\rangle$, and $b_{\downarrow}^{\dagger}|0\rangle$ are involved in the representation. We argue that this overcounting of the states does not affect the physics. The partition function being a trace, it is the same when we evaluate it on the fermionic or the bosonic subspace separately. Then on the extended subspace, it just acquires a multiplicative factor that will cancel in the calculation of any physical quantity. One can easily check that the representation that we propose satisfies the standard rules of $S U(2)$ algebra.

Let us consider the three-dimensional KLM near half filling $\left(n_{c} \leqslant 1\right)$. The Hamiltonian is

$$
H=\sum_{k \sigma} \varepsilon_{k} c_{k \sigma}^{\dagger} c_{k \sigma}+J \sum_{i} \mathbf{S}_{\mathbf{i}} \cdot \mathbf{s}_{i}
$$

where $J(>0)$ is the Kondo interaction and $\mathbf{s}_{i}$ $=\Sigma_{\sigma \sigma^{\prime}}\left(c_{i \sigma}^{\dagger} \tau_{\sigma \sigma^{\prime}} c_{i \sigma^{\prime}}\right)$ is the spin of conduction electrons. In 
the representation introduced before, the partition function can be written as the following path integral

$$
\begin{aligned}
& Z=\sum \int \mathcal{D} c_{i \sigma} \mathcal{D} f_{i \sigma} \mathcal{D} b_{i \sigma} d \lambda_{i} \\
& \quad \times \exp \left\{-\int_{0}^{\beta} d \tau\left[\mathcal{L}(\tau)+\mathcal{H}+\sum_{i} \lambda_{i}\left(n_{f_{i}}+n_{b_{i}}-1\right)\right]\right\} \\
& \text { with } \mathcal{L}(\tau)=\sum_{i \sigma}\left(c_{i \sigma}^{\dagger} \partial_{\tau} c_{i \sigma}+f_{i \sigma}^{\dagger} \partial_{\tau} f_{i \sigma}+b_{i \sigma}^{\dagger} \partial_{\tau} b_{i \sigma}\right) \\
& \text { and } \mathcal{H}=\sum_{k \sigma} \varepsilon_{k} c_{k \sigma}^{\dagger} c_{k \sigma}+J \sum_{i}\left(\mathbf{S}_{f_{i}}+\mathbf{S}_{b_{i}}\right) . \mathbf{s}_{i}-\mu \sum_{i} n_{c_{i}} .
\end{aligned}
$$

The time-independent Lagrange multiplier $\lambda_{i}$ is introduced to enforce the local constraint $n_{f_{i}}+n_{b_{i}}-1=0$.

Performing a Hubbard-Stratonovich transformation and neglecting the space and time dependence of the fields in a self-consistent saddle-point approximation, we have

$$
\begin{gathered}
Z=\sum \int d \eta d \eta^{*} \mathcal{C}\left(\sigma_{0}, \epsilon_{f}, \eta, \eta^{*}\right) Z\left(\eta, \eta^{*}\right), \\
Z\left(\eta, \eta^{*}\right)=\sum_{\sigma} \int \mathcal{D} c_{i \sigma} \mathcal{D} f_{i \sigma} \mathcal{D} b_{i \sigma} \\
\times \exp \left\{-\int_{0}^{\beta} d \tau\left[\mathcal{L}(\tau)+\mathcal{H}^{\prime}\right]\right\} \\
\text { with } \begin{array}{c}
\mathcal{H}^{\prime}=\sum_{k}\left(f_{k \sigma}^{\dagger}, c_{k \sigma}^{\dagger}, b_{k \sigma}^{\dagger}\right) H_{0}\left(\begin{array}{c}
f_{k \sigma} \\
b_{k \sigma}
\end{array}\right), \\
H_{0}=\left(\begin{array}{ccc}
\varepsilon_{f} & \sigma_{0} & 0 \\
\sigma_{0} & \varepsilon_{k} & \eta \\
0 & \eta^{*} & \varepsilon_{f}
\end{array}\right),
\end{array}
\end{gathered}
$$

where $\mathcal{C}\left(\sigma_{0}, \epsilon_{f}, \eta, \eta^{*}\right)$ is an integration constant. $\varepsilon_{f}$ is the saddle-point value of the Lagrange multiplier $\lambda_{i}$. Note the presence of a Grassmannian coupling $\eta$ between $c_{i \sigma}$ and $b_{i \sigma}$, in addition to the usual coupling $\sigma_{0}$ between $c_{i \sigma}$ and $f_{i \sigma}$ responsible for the Kondo effect. $H_{0}$ is of the type

$$
\left(\begin{array}{ll}
a & \sigma \\
\rho & b
\end{array}\right)
$$

in which $a, b(\rho, \sigma)$ are matrices consisting of commuting (anticommuting) variables. Note the supersymmetric structure of the matrix $H_{0}$ similar to the supermatrices appearing in the theory of disordered metals. ${ }^{5}$

$H_{0}$ being hermitian, the matrix $U^{\dagger}$ transforming the original basis $\psi^{\dagger}=\left(f^{\dagger}, c^{\dagger}, b^{\dagger}\right)$ to the basis of eigenvectors $\Phi^{\dagger}$ $=\left(\alpha^{\dagger}, \beta^{\dagger}, \gamma^{\dagger}\right)$ is unitary $\left(U U^{\dagger}=U^{\dagger} U=1\right) . \Phi^{\dagger}=\psi^{\dagger} U^{\dagger}$ with $U^{\dagger}$ a supersymmetric matrix. $\alpha^{\dagger}$ and $\beta^{\dagger}$ are the fermionic eigenvectors whose eigenvalues, determined from $\operatorname{det}[(a$ $\left.-E)-\sigma(b-E)^{-1} \rho\right]=0$, are

$$
E_{\mp}=\frac{\left(\varepsilon_{k}+\varepsilon_{f}\right) \mp \sqrt{\left(\varepsilon_{k}-\varepsilon_{f}\right)^{2}+4\left(\sigma_{0}^{2}+\eta \eta^{*}\right)}}{2} .
$$

$\gamma^{\dagger}$ is the bosonic eigenvector whose eigenvalue, determined from $\operatorname{det}\left[(b-E)-\rho(a-E)^{-1} \sigma\right]=0$ is $E_{\gamma}=\varepsilon_{f}$.

In the scheme we propose, $\sigma_{0}$ and $\epsilon_{f}$ are slow variables that we determine by solving saddle-point equations, while $\eta, \eta^{*}$ are fast variables defined by a local approximation. As we will see, the latter approximation incorporates part of the fluctuation effects. Indeed, performing the functional integration of Eq. (6) over the fermion and boson fields ${ }^{5}$ yields a superdeterminant (S Det) form written as follows:

$$
Z\left(\eta, \eta^{*}\right)=\mathrm{S} \operatorname{Det}\left(\partial_{\tau}+H_{0}\right)
$$

where $\quad \operatorname{Set}\left(\partial_{\tau}+H\right)=\frac{\operatorname{Det}\left(G^{-1}-\sigma D \rho\right)}{\operatorname{Det}\left(D^{-1}\right)}$,

$$
G^{-1}=\partial_{\tau}+a \text { and } D^{-1}=\partial_{\tau}+b .
$$

Expanding to second order in $\eta, \eta^{*}$ allows us to define the propagator $G_{\eta \eta^{*}}\left(\mathbf{k}, i \omega_{n}\right)$ associated to the Grassmann variable $\eta$ and hence, the closure relation for $x_{0}^{2}=\left\langle\eta \eta^{*}\right\rangle$

$$
\begin{aligned}
& x_{0}^{2}=\frac{1}{\beta} \sum_{\mathbf{k}, i \omega_{n}} G_{\eta \eta^{*}}\left(\mathbf{k}, i \omega_{n}\right), \\
& \text { with } \quad G_{\eta \eta^{*}}\left(\mathbf{k}, i \omega_{n}\right)=\frac{J}{\left[1-J \Pi_{c b}^{0}\left(\mathbf{k}, i \omega_{n}\right)\right]} \\
& \text { and } \quad \prod_{c b}^{0}\left(\mathbf{k}, i \omega_{n}\right)=\frac{1}{\beta} \sum_{\mathbf{q}, i \omega_{n}} G_{c c}\left(\mathbf{k}+\mathbf{q}, i \omega_{n}\right. \\
& \left.+i \omega_{\nu}\right) D\left(\mathbf{q}, i \omega_{n}\right) .
\end{aligned}
$$

Contrary to Ref. 6 which assumes $x_{0}^{2}=0$ leading to a twofluid model description, the closure Eq. (8) that we introduce defines a finite $x_{0}^{2}$. This parameter $x_{0}^{2}$ plays a major role in controlling the relative weights of fermion and boson statistics.

The resolution of the saddle-point equations, keeping the number of particles conserved, leads to

$$
\begin{gathered}
y_{F}=-D \exp \left[-1 /\left(2 J \rho_{0}\right)\right], \\
1=\frac{2 \rho_{0}\left(\sigma_{0}^{2}+x_{0}^{2}\right)}{-y_{F}}, \\
\mu=-\frac{\left(\sigma_{0}^{2}+x_{0}^{2}\right)}{D}, \\
\lambda_{0}=0,
\end{gathered}
$$

where $y_{F}=\mu-\varepsilon_{f}$ and $\rho_{0}=1 / 2 D$ is the bare density of states of conduction electrons. From that set of equations, we find $\varepsilon_{f}=0$.

The resulting spectrum of energies is schematized in Fig. 1. At zero temperature, only the lowest band $\alpha$ is filled with an enhancement of the density of states at the Fermi level (and hence of the mass) unchanged from the standard slaveboson theories $\left[\rho\left(E_{F}\right)\right] / \rho_{0}=1+\left(\sigma_{0}^{2}+x_{0}^{2}\right) / y_{F}^{2}=1+D /$ $\left(-y_{F}\right) \gg 1$. This large mass enhancement is related to the flat part of the $\alpha$ band associated with the formation of the Abrikosov-Suhl resonance pinned at the Fermi level. While this feature was already present in the purely fermionic de- 


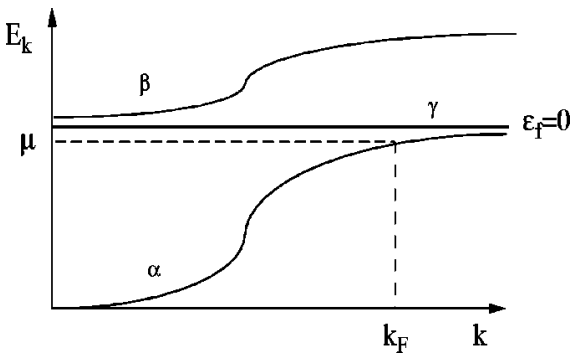

FIG. 1. Sketch of energy versus wave number $k$ for the three bands $\alpha, \beta$, and $\gamma$ resulting of the diagonalization of supersymmetric $H_{0}$.

scription, ${ }^{3,4}$ it is to be noted that the formation of a dispersionless bosonic band within the hybridization gap is an entirely new result of the theory.

The relative weight of boson and fermion statistics in the spin representation is related to $x_{0}^{2}: n_{b} / n_{f}=x_{0}^{2} / \sigma_{0}^{2}$. It is then interesting to follow the $J$ dependence of $x_{0}^{2}$ as determined by the closure Eq. (8). The result is reported in Fig. 2. This bell-shaped curve can be interpreted in the light of the exhaustion principle mentioned in the introduction. In the limit of large $J$, the Kondo temperature-scale $T_{K}=D \exp$ $\left[-1 /\left(2 J \rho_{0}\right)\right]$ is of order of the bandwidth. One then expects a complete Kondo screening without any residual unscreened spin degrees of freedom. It is then natural to derive a zero value of $x_{0}^{2}$ and hence, of $n_{b}$. The opposite limit at small $J$ corresponds to the free case of uncoupled impurity spins and conduction electrons. It also leads to $x_{0}^{2}=0$. The finite value of $x_{0}^{2}$ between these two limits with a maximum reflects the incomplete Kondo screening effect in the Kondo lattice, the unscreened spin degrees of freedom being described by bosons.

Largely discussed in the litterature ${ }^{8}$ is the question concerning the Fermi-surface sum rule: do the localized spins of the Kondo lattice contribute to the counting of states within the Fermi surface or do they not? Depending on the answer, one expects large or small Fermi surfaces. The supersymmetric theory leads to a firm conclusion in favor of the former. One can check that the number of states within the Fermi surface is just equal to $n_{c}+n_{b}+n_{f}$, i.e., $n_{c}+1$. The Fermisurface volume includes a contribution of one state per localized spin in addition to that of conduction electrons. ${ }^{1}$ This conclusion that appears reasonable if one recalls that the

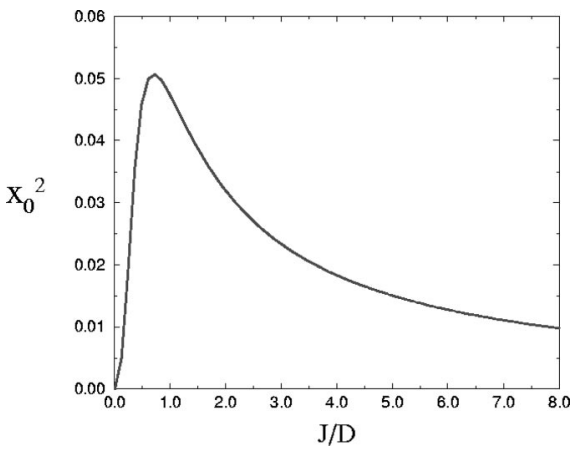

FIG. 2. $J / D$ dependence of the coupling $x_{0}^{2}=\left\langle\eta \eta^{*}\right\rangle$ fixing the relative weight of fermion and boson statistics. The unit on the vertical axis is $D^{2}$

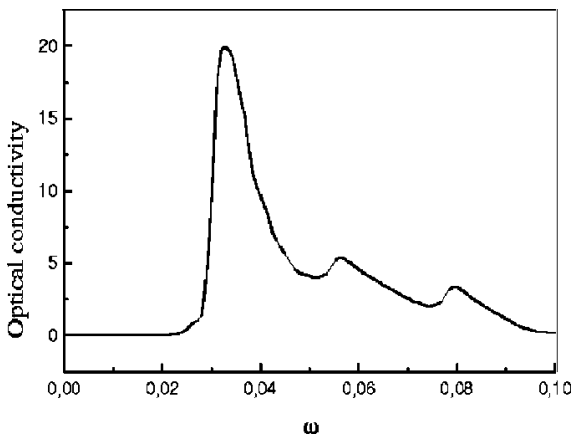

FIG. 3. Frequency dependence of the optical conductivity $\sigma(\omega)$ at $T=0$ for $D=0.8$ and $T_{K}=0.001$.

KLM is an effective Hamiltonian derived from the periodic Anderson model, has been reached before by several other authors. ${ }^{8,9} \mathrm{We}$ do think that it is a good sign to recover previously established results if they are correct.

Let us now consider the response functions to some external fields namely the dynamical spin susceptibility $\chi^{a b}(\mathbf{q}, \omega)$ and the frequency-dependent optical conductivity $\sigma^{a b}(\omega)(a, b=x, y, z)$. For that purpose, we introduce the Matsubara correlation functions associated with the operator $\mathcal{O}^{a}(\mathbf{q}, \tau): \chi^{a b}\left(\mathbf{q}, i \omega_{\nu}\right)=\int_{0}^{\beta} d \tau \exp ^{i \omega_{\nu} \tau}\left\langle T_{\tau} \mathcal{O}^{a}(\mathbf{q}, \tau) \mathcal{O}^{b}(-\mathbf{q}, 0)\right\rangle$. The operator related to the spin-spin correlation function is the $a$ component of the spin expressed in the mixed representation introduced in the paper by: $S^{a}(\mathbf{q})$ $=\Sigma_{k, \sigma, \sigma^{\prime}} f_{k+q, \sigma}^{\dagger} \tau_{\sigma \sigma^{\prime}}^{a} f_{k, \sigma^{\prime}}+b_{k+q, \sigma}^{\dagger} \tau_{\sigma \sigma^{\prime}}^{a} b_{k, \sigma^{\prime}}$. As usual, the dynamical spin susceptibility is then derived from the spinspin correlation function by the analytical continuation $i \omega_{\nu}$ $\rightarrow \omega+i 0^{+}$. In the same way, the operator related to the current-current correlation function is the $a$ component of the $c$ current. In the case of a cubic lattice: $J_{c}^{a}(\mathbf{q})$ $=2 \Sigma_{k, \sigma, \sigma^{\prime}} \sin k_{a} c_{k+q, \sigma}^{\dagger} c_{k, \sigma}$. The frequency-dependent optical conductivity is then obtained from the current-current correlation function by the analytical continuation following: $\sigma^{a b}(\omega)=\left[\chi^{a b}\left(\mathbf{q}, \omega+i 0^{+}\right)-\chi^{a b}\left(\mathbf{q}, i 0^{+}\right)\right] i \omega$.

By expanding the previous expressions in the basis of the eigenstates $\left(\alpha^{\dagger} \beta^{\dagger} \gamma^{\dagger}\right)$ of $H_{0}$, we have computed the frequency dependence of $\chi^{a b}(\mathbf{Q}, \omega)$ at the antiferromagnetic wave vector $\mathbf{Q}$ and $\sigma^{a b}(\omega)$ at zero temperature. The two response functions show very different frequency dependence. The frequency scale at which the dynamical spin susceptibility takes noticeable values is much smaller than for the optical conductivity. This can be understood in the following way. The bosonic $\gamma$ band is called to play a role only when spin is concerned namely for the dynamical spin susceptibility. That feature comes from the fact that the spin is related to both fermionic and bosonic operators while the $c$ current is simply expressed within fermionic operators. Therefore, one can show that the dynamical spin susceptibility involves transitions between all three bands $\alpha, \beta$, and $\gamma$. The main contribution for $\chi^{a b}(\mathbf{Q}, \omega)$ is due to the particlehole pair excitations from the fermionic $\alpha$ to the bosonic $\gamma$ band. Oppositely, the optical conductivity is associated with transitions between fermionic bands only. As can be seen in Fig. 3, a gap appears in the frequency dependence of $\sigma(\omega)$ equal to the direct gap between the $\alpha$ and $\beta$ bands. The latter 
result agrees with the predictions of the dynamical meanfield theory in the limit of infinite dimensions. ${ }^{12}$ The whole discussion clarifies the physical content of the novel bosonic mode brought by the supersymmetric approach. That mode is related to the spin excitations. It introduces new features in the dynamical spin susceptibility by comparison to the standard slave-boson theories while it does not affect the optical conductivity.

In summary, we have shown that the supersymmetric method proves to be a powerful tool to account for both
Fermi-liquid excitations and residual spin degrees of freedom in heavy-Fermion systems. The work opens the way for further investigations as the systematic study of the effects of fluctuations in the pure Kondo lattice model or the incorporation of additional RKKY interactions in an extended Kondo lattice model.

We would like to thank P. Coleman, F. Delduc, A. Georges, P.A. Lee, M.J. Rozenberg, A. Yashenkin, and T. Ziman for very helpful discussions. M.L. is a member of the Centre National de la Recherche Scientifique (CNRS).

${ }^{7}$ S. Doniach, Physica B \& C 91, 231 (1977).

${ }^{8}$ H. Tsunetsugu, M. Sigrist, and K. Ueda, Rev. Mod. Phys. 69, 809 (1997), and references therein.

${ }^{9}$ R.M. Martin, Phys. Rev. Lett. 48, 362 (1982).

${ }^{10}$ C. Lacroix, and M. Cyrot, Phys. Rev. B 20, 1969 (1979).

${ }^{11}$ N. Read, D.M. Newns, and S. Doniach, Phys. Rev. B 30, 3841 (1984).

${ }^{12}$ M.J. Rozenberg, G. Kotliar, and H. Kajueter, Phys. Rev. B 54, 8452 (1996). 